\title{
LAS SALINAS DE LA BAHÍA DE CÁDIZ DURANTE LA ANTIGÜEDAD: VISIÓN GEOARQUEOLÓGICA DE UN PROBLEMA HISTÓRICO*
}

\author{
THE SALT MINES OF THE BAY OF CÁDIZ DURING THE ANTIQUITY: \\ GEOARCHAEOLOGICAL VIEW OF A HISTORIC PROBLEM
}

\author{
por \\ Carlos alonso Villalobos ${ }^{* *}$ \\ F. JAVIER GRACIA PRIETO ${ }^{* * *}$ \\ LOÏC MÉNANTEAU ${ }^{* * * *}$
}

RESUMEN

\begin{abstract}
A pesar de su importancia histórica como elemento de primera necesidad, la sal, junto a todo el patrimonio material a ella asociada, es una de las grandes desconocidas. Si bien la investigación histórico-arqueológica ha desvelado durante las últimas décadas muchas de las incógnitas relativas a la producción y comercialización de la sal gema y de ignición tanto en latitudes de la Europa septentrional como meridional, no existían hasta ahora huellas materiales de la producción del cloruro sódico durante la antigüedad por medio de sistemas de evaporación solar. El análisis geoarqueológico del medio costero de San Fernando (Bahía de Cádiz) ha permitido, en parte, restituir el paleopaisaje marismeño de el saco interior de la bahía gaditana, identificando restos de las estructuras de antiguas salinas romanas y dejando ver la necesidad de reinterpretar muchos indicadores que, relacionados con esta actividad, permanecían mal interpretados.
\end{abstract}

ABSTRACT Despite its historic importance as an essential product, our knowledge of salt, as well as all the material elements associated with it, is very limited. Although over the last few decades historic and archaeological research has cleared up numerous obscure points regarding the production of and trade in rock and igneous salt in both Northern and Southern Europe, until recently there were no physical traces of the production of salt by natural evaporation in Antiquity. The geoarchaeological analysis of the littoral of San Fernando (Bay of Cadiz) has permitted at least a partial reconstruction of the marshland paleolandscape of the inner bay and the identification of vestiges of Roman saltpans. It is also clear that many of the signs relating to this activity, hitherto misinterpreted, will have to be reviewed.

\footnotetext{
* Este trabajo es una aportación al Proyecto BTE2003-05706 del Ministerio de Ciencias y Tecnología-FEDER, y constituye también una aportación al Grupo de Investigación RNM328 del PAI Junta de Andalucía.

** Instituto Andaluz del Patrimonio Histórico. Consejería de Cultura. Junta de Andalucía. cayac@arrakis.es

*** Dpto. de Ciencias de la Tierra. Facultad de Ciencias del Mar y Ambientales. Universidad de Cádiz. javier.gracia@uca.es **** Laboratoire Géolittomer-Nantes. LETG-UMR 6554. Centre National de la Recherche Scientifique et Université de Nantes. Francia. loic.menanteau@humana.univ-nantes.fr
} 
Palabras claves Sal; Salinas; Salazones; Geoarqueología; Paleotopografía; Bahía de Cádiz

Key words

Salt; Salt mine; Salted fish; Geoarchaeology; Palaeotopography; Bay of Cádiz

\section{INTRODUCCIÓN}

De la gran diversidad de sales minerales existentes en la naturaleza, sin duda la más conocida y utilizada es la sal común o cloruro sódico. Por ser un elemento imprescindible para el normal desarrollo de la actividad celular y orgánica de los seres vivos, este mineral ha tenido a lo largo de la historia una gran relevancia, encontrándose presente en todas las culturas.

Tras la revolución neolítica, la alimentación basada en la consumo de proteínas animales de los antiguos grupos de cazadores-recolectores fue cediendo terreno a un nuevo tipo de dieta en el que los cereales están cada vez más presentes. Esto produjo una notable reducción del aporte de sales, deficiencia que debió suplirse desde entonces mediante el consumo directo de sal común.

El cloruro sódico, igualmente imprescindible para la correcta alimentación del ganado herbívoro rumiante, se encontraba también presente en ámbitos de la vida como la conserva de carnes y pescado, el curtido de pieles, la curación de heridas en personas y animales, etc., llegando a formar parte por su importancia de muchas ceremonias y ritos religiosos. Controlar su producción y distribución llegó a ser por ello un objetivo primordial para los humanos desde la más remota antigüedad, motivo por el que reyes y gobernantes desde la prehistoria buscaron mecanismos para hacerse con el monopolio de su producción y comercialización, convirtiéndola en un codiciado objeto de alto valor. Su comercio, como el del estaño, las especias o la seda, abrió grandes rutas de exportación, siendo transportada por los mercaderes a miles de kilómetros, atravesando áridos desiertos y cruzando peligrosos mares, hasta alcanzar los rincones más apartados del mundo.

\section{VISIÓN ARQUEOLÓGICA DE LA PRODUCCIÓN DE SAL}

En Europa, los primeros datos relativos a la explotación y el comercio del denominado "oro blanco" vienen de la mano de la arqueología prehistórica. En la Península Balcánica (Tasic 2002); Hallstat (Austria) y en otros lugares de la Europa central como Polonia, Alemania, etc. (Weller 2002b; Saille 2002), se han encontrado evidencias de la producción y el comercio de la sal gema y de ignición con anterioridad al $4000 \mathrm{a}$. C.

En las costas bretonas francesas se ha podido documentar una fuerte concentración y variedad tipológica de talleres para la extracción de sal mediante técnica de evaporación por ignición (las briqueries) (Véase al respecto Gouletquer y Kleinmann 1972; Lemonnier 1984a y 1984b; Daire 1994; y como síntesis y recopilación bibliográfica, Cassen 2001; Fernández 2000 y Weller 2002a), actividad extractiva también constatada recientemente en Pisa, Italia (Pascuaquinucci y Menchelli 2002); los Alpes (Morin 2002) y el Pirineo francés (Chopin 2002); la Marismilla (Puebla del Río, Sevilla) (Escacena et al. 1988, 1994 y 1996) y la desembocadura del río Guadiaro (San Roque, Cádiz) (Schubart 1986), yacimientos estos últimos que vienen a confirmar la producción de sal ígnea en nuestra tierra entre el 4000 a. C. y el siglo VIII a.C. Trabajos recientes como el de S. Cassen, están permitiendo obtener mayor información sobre algunas de estas salinas neolíticas (la Marismilla), e identificar para ese mismo momento cultural nuevos enclaves de producción de sal hasta el momento no interpretados como tales en el Puerto de Santa María (Cádiż)(Cassen 2001: 258).

Las primeras referencias clásicas al respecto de las salinas de evaporación solar las encontramos en Tito Livio (Historia romana, 1-33) y en Plinio (Historia Natural, XXXI-41). Según ellos Anco Marcio 
(641-616 a. C.), fundador del puerto de Ostia, roturó en las marismas del delta del Tíber las salinas que aprovisionaban a Roma, a través de la que posteriormente se denominaría Vía Salaria.

En la sociedad preindustrial, tal como escribiera Plinio el Viejo (H.N., XXXI, 88), no era posible concebir una vida civilizada sin sal. Era imprescindible ser autosuficientes en lo relativo a la alimentación, almacenamiento de mercancías en previsión de momentos de malas cosechas o catástrofes. Las salazones en este sentido garantizaban el abastecimiento de la población y los ejércitos en cualquier lugar y momento del año. Carnes y pescados salados han sido, pues (y aún continúan siéndolo para buena parte de la población de la Tierra), la base del aporte de proteínas en el mundo occidental. Por ello, la búsqueda de nuevos y mejores métodos para la producción y el control de comercio de la sal debió ser una constante histórica. Por Livio (Historia romana, II-9) sabemos que los romanos, en los primeros años de la República, dictaron normas encaminadas a ejercer el monopolio estatal sobre la producción de la sal, con el fin de asegurar el abastecimiento de la población y evitar el alza de los precios. Según el Digesto (Dig. 50.15.4; 27.9.5; 33.2.32; 50.16.17) durante el Imperio el Estado ejercía el monopolio sobre la venta de la sal, controlando el precio y las condiciones para su compra-venta; pero no controlaba la fabricación de ésta y la explotación de las salinas (Pastor 1880). Estas últimas estaban, por tanto, en manos de particulares, que eran libres para explotarlas directamente, arrendarlas a terceros o incluso hacer transmisión de ellas por medio de su venta. Se les exigía, eso sí, que fuesen inscritas por sus propietarios legales en un censo oficial (Dig. 50.15.4). Así lo viene a confirmar el hallazgo en Egipto de un papiro del periodo ptolemaico en el que se cita a los salinatores de la antigua ciudad de Tebtunis agrupados en sociedades para reglamentar las condiciones de la venta y recaudación de los impuestos estatales sobre la sal (Boak 1937). Durante este periodo el Estado controlaba los beneficios obtenidos de la producción de sal por medio, tanto de la promulgación de normativas específicas de venta, como de la implantación de un precio estable para ella. Estas condiciones no debieron ser las mismas en todo el Imperio. Cada localidad se sometía a reglamentaciones particulares que, en algunos casos, podían llegar incluso a prohibirles la explotación de sus propias salinas, obligando a sus habitantes a adquirir la sal necesaria para su consumo en lugares concretos y a precios estipulados.

A pesar de su importancia histórica como elemento de primera necesidad, la sal, junto a todo el patrimonio material a ella asociada, sigue siendo una de las grandes olvidadas, motivo por el que incluso en la actualidad todo el patrimonio salinero de nuestro litoral se encuentra, a pesar de su indudable interés histórico-etnográfico, en lamentable estado de abandono y progresivo deterioro. La parquedad de las fuentes de información históricas referidas a su explotación y comercio (especialmente con anterioridad al año mil) y la falta de sensibilidad respecto al tema por parte del mundo de la investigación histórico-arqueológica, han contribuido en parte a ello.

\section{CÁDIZ Y LA SAL}

En las últimas décadas la investigación histórico-arqueológica en el marco de la bahía gaditana está permitiendo un mejor conocimiento de la actividad pesquera y conservera. A saber: tipos de especies capturadas; técnicas empleadas para ello; complejos industriales de transformación; composición de los productos elaborados; envases empleados para el transporte; ruta de comercialización; etc. (véase una síntesis al respecto en García y Muñoz 2003). Sin embargo la sal, sustancia básica para el proceso de elaboración de estas conservas, sigue siendo la gran olvidada, desconociéndose prácticamente todo en relación con los métodos, técnicas y zonas de producción.

Según relata Estrabon (III, 5, 11), los fenicios de Gadir comerciaban con las Cassiterides cambiando plomo y estaño por sal y diversos productos manufacturados, dando testimonio de que, desde fecha temprana, la producción y comercialización de sal y salazones era una de las principales actividades económicas para 
estas comunidades humanas. A partir de los siglos $\mathrm{V}$ y IV a. C., las conservas saladas que se producían en el Circulo del Estrecho (las afamadas salazones y salsas de pescado gaditanas) experimentaron un fuerte auge, exportándose incluso a la ciudad de Atenas (Frutos y Muñoz 1996; García y Ferrer 2001). En ese momento, según apunta Manfredi (1992), parece observarse en el Mediterráneo una clara organización, estructuración y control del comercio de la sal por parte del Estado, en estrecho vínculo con el culto y los santuarios a Melqart.

A partir del siglo III, tras el mantenimiento de la actividad salazonera gaditana a lo largo de toda la antiguiedad (al menos entre los siglos VI a. C. y II d.C.), el sector entró en crisis reduciéndose notablemente la producción y arrastrando tras de sí al resto de las actividades implicadas.

Hasta ese momento, ni las fuentes escritas, ni la arqueología, habían aportado información concreta relativa a los métodos empleados por los gaditanos para la obtención de la sal destinada al consumo local y a la fuerte demanda del sector salazonero. Habrá que esperar hasta los siglos XIII y XIV para encontrar en las primeras crónicas medievales (Franco $s$. a.) referencia a salinas de evaporación solar sobre marismas cuya actividad se asociaba, una vez más, a las crecientes demandas del pujante sector pesquero.

¿Cuáles eran pues los sistemas utilizados para la obtención de sal marina con anterioridad al siglo XIII? Pensamos que sólo desde el conocimiento de los diferentes sistemas de producción empleados históricamente para la obtención de sal, y del análisis geoarqueológico y paleoambiental del medio sobre el que implantaron esas salinas, es posible localizar y reconocer los escasos restos materiales que se puedan conservar de sus estructuras. Para ello comenzaremos por realizar un breve análisis de los principales métodos y técnicas utilizados para la producción de la sal marina.

\section{MÉTODOS Y TÉCNICASPARA LA EXTRACCIÓN DE LA SAL MARINA: DE LA IGNICIÓN A LA EVAPORACIÓN POR INSOLACIÓN}

Los sistemas empleados para la obtención de sal pueden variar en función de las características geológicas y climáticas de la zona (Cuadro I).

Sabemos que, en otras latitudes, desde tiempos antiguos y con anterioridad a la aparición de los modelos de salinas de evaporación solar, la sal se obtenía principalmente por dos sistemas:

$>$ La explotación de vetas de cloruros, originadas bien por la desecación de antiguas superficies de agua marina o por afloramiento diapírico, dando lugar a la denominada sal gema ${ }^{1}$.

$>$ Por la evaporación del agua salobre mediante calentamiento ígneo, hasta la cristalización de los cloruros ${ }^{2}$.

En nuestras tierras, al menos hasta el Bronce Final, a sal debió obtenerse por calentamiento ígneo del agua y cuerpos salinos, técnica ampliamente documentada arqueológicamente en la costa atlántica francesa hasta época galorromana (Weller, 2002a).

En un momento aún por determinar, un nuevo método de producción más intensivo y rentable, en el que la cristalización del cloruro sódico se alcanzaba sustituyendo la tradicional fuente de calor ígnea por la solar, vendría a sustituir al anterior (al menos en zonas con óptimas condiciones de insolación y precipitaciones como el litoral de Cádiz). Sabemos que éste ya era conocido en el siglo I, si bien su origen es muy anterior, según señala el geógrafo y naturalista Plinio en el libro XXXI de su Naturalis Historia. En dicha obra Plinio

1. En la obra de autores de la Antigüedad como Plinio, Aulo Gelio, Estrabón, Columela, Paladio, San Isidoro de Sevilla, etc encontramos las primeras referencias a la producción de la sal en la Península. Véanse al respecto los trabajos de Morère (1994) y Fernández (2000).

2. Durante este proceso, el agua salina era calentada en recipientes cerámicos hasta su cristalización total, obteniéndose con ello unos bloques de sal (más aptos para el transporte y comercio) a los que genéricamente se les denomina "pan de sal". 
establece una clara diferencia entre la que denomina sal nativa (XXXI, 73 a XXXI, 80), procedente de lagunas, montañas, ríos, fuentes y la espuma dejada por el mar sobre la orilla, y la sal artificial (XXXI, 81 a XXXI, 83), obtenida principalmente mediante la antropización de marismas costeras, fuentes y manantiales salinos. Por la descripción que nos facilita Rutilius Namatianus (De reditu suo, I, 475-478) de una salina próxima a Roma, conocemos que la técnica empleada entonces difería poco de la de nuestros denominados sistemas extractivos tradicionales. Las exigencias ambientales y características técnicas de este nuevo método hicieron desde entonces de la extracción de sal una actividad estacional que, al menos en el marco gaditano, se inicia con la primavera y finaliza a la llegada del otoño, curiosamente en paralelo al proceso migratorio (de ida y vuelta) de los túnidos por nuestras costas.

\section{Cuadro I.}

Diferente sistemas productivos para la obtención de la sal (adaptado de Weller, 2002b)

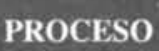

PRODUCTIVO

MATERIAS PRIMAS / PROCEDIMIENTOS/ PRODUCTOS

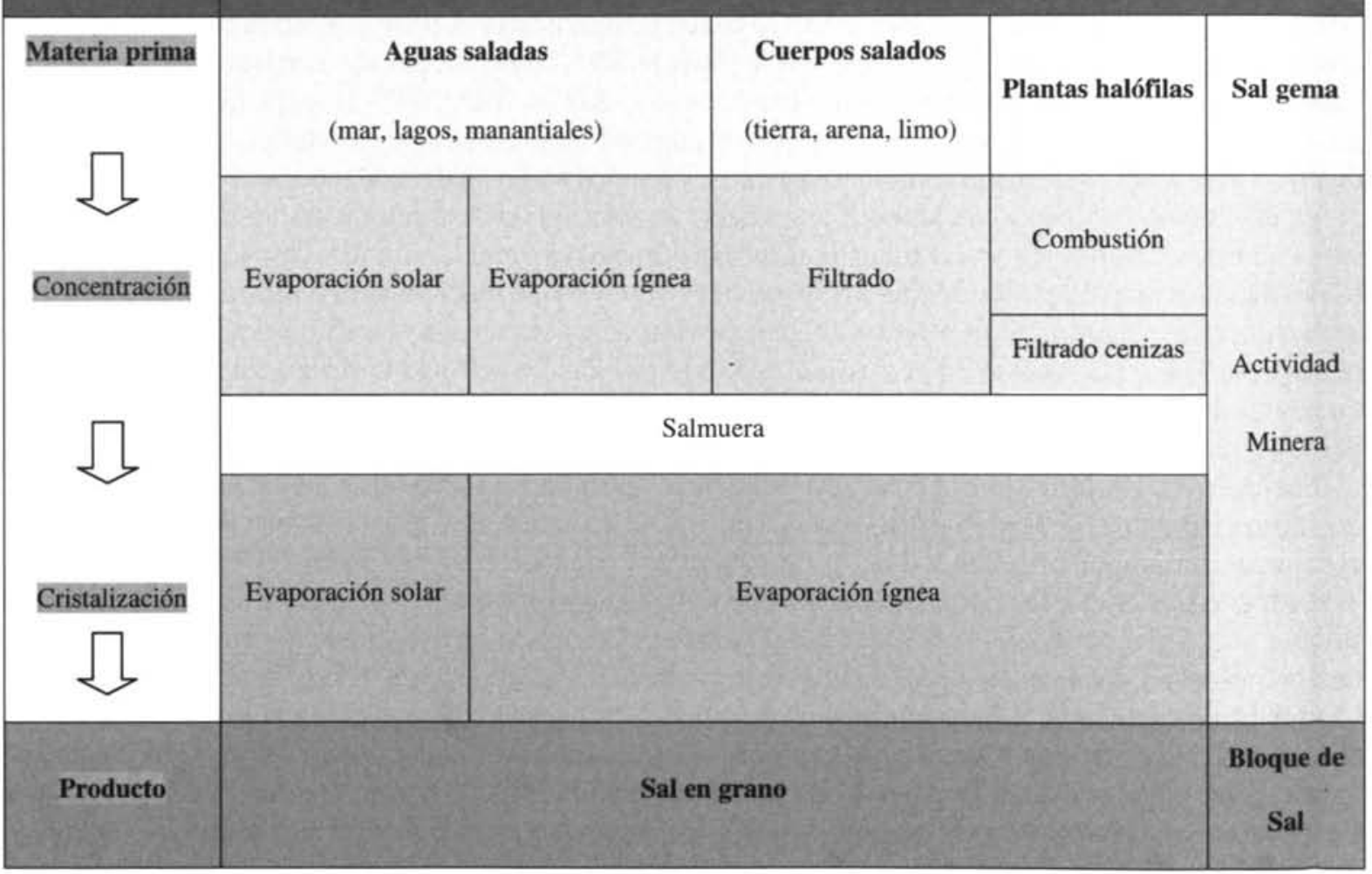

La forma, el tamaño y la topografía de estas salinas de evaporación por insolación se ven condicionadas, principalmente, por las características del sustrato sobre el que se implantan (roca o fango) y por las condiciones climáticas de la zona. Con el paso de los siglos este método extractivo se ha ido perfeccionando para aumentar la velocidad de evaporación en función del nivel de insolación, pluviosidad, régimen de vientos, etc. de cada zona. No obstante, todas las salinas disponen de una estructura similar, en la que se diferencian tres tipos de áreas o dependencias: 
- Los depósitos de almacenamiento donde se acumula el agua salobre que alimenta la salina.

- Las zonas de evaporación, en las que se retiene el agua mientras es sometida a un proceso de evaporación y concentración salina por exposición solar.

- Los cristalizadores, zona en la que, por los mismos procedimientos, se alcanza el nivel de máxima concentración salina, cristalizando los cloruros.

Según el tipo de explotación y la técnica empleada para la misma, la circulación hídrica entre cada uno de estos espacios se activa por procedimientos naturales (la propia gravedad) o mediante el empleo de fuerza animal o mecánica (desde la propia mano del hombre, especialmente en el caso de las salinas artesanales sobre roca, hasta el uso de norias y sistemas de bombeo activados tradicionalmente por energía eólica o animal). De entre todas ellas destacan por la sencillez y escasa tecnificación de su sistema extractivo las salinas marítimas sobre roca, en algunos casos conformadas por simples estructuras de cristalizadores generadas mediante la mínima transformación de las charcas naturales del terreno.

Precisamente en relación con esta tipología, queremos traer a colación una serie de indicadores arqueológicos existentes a lo largo del litoral de Cádiz -en Chipiona (Ramos y Riesco 1983), y en Caños de Meca, Barbate- cuyo paralelo tipológico más próximo lo encontramos sin duda en antiguas salinas sobre roca del Mediterráneo oriental (Fig. 1) y Canarias (González 1996) ${ }^{3}$. Consisten en una serie de pocetas circulares de aproximadamente $1 \mathrm{~m}$ de diámetro y $15-20 \mathrm{~cm}$ de profundidad excavados sobre la plataforma rocosa costera y que, hasta el momento, venían siendo interpretados como huellas de antiguas explotaciones de canteras para la extracción de piedras de molinos (Ramos y Riesco, 1983: 387). La escasa dureza y compactación del basamento de arenisca ostionera sobre el que está tallada esta supuesta cantera hace poner en tela de juicio el hipotético uso de los productos pétreos obtenidos como piedras de molino. Pensamos que, por paralelos tipológicos, estas huellas podrían ponerse en relación con estructuras de cristalizadores para la extracción de sal marina por procedimientos de insolación. Su proximidad en ambos casos a complejos industriales de salazones de pescado de época romana viene a apoyar esta función. Su actual posición intermareal, inundados en situación de pleamar, entra en aparente contradicción con una y otra hipótesis, a no ser, tal como hemos podido apreciar mediante diversos indicadores arqueológicos detectados a lo largo de esta franja litoral, que durante el momento en el que el ser humano actuó sobre este basamento rocoso la posición relativa del nivel del mar se encontraba ligeramente por debajo del actual (Gracia et al. 1995; Sainz 2003)

Sin lugar a dudas, el máximo exponente de la intervención humana para la obtención de la sal lo constituyen las salinas labradas sobre barro, especialmente en la costa atlántica, donde la presencia de mareas permite el desarrollo de amplias marismas. Este tipo de salinas de evaporación puede ser descrito como una sucesión de recintos estancos dispuestos cada vez a menor cota para favorecer, a través de ellos, la circulación del agua salobre por la simple acción de la gravedad. Durante su recorrido, por efecto de la evaporación, la concentración salina va aumentado progresivamente hasta alcanzar, finalmente, la precipitación en los cristalizadores de cloruro sódico. Estas salinas pueden adoptar diferentes tipologías en función de las condiciones climáticas de la zona, tamaño, topografía y tipo de sustrato sobre el que se implantan, pero genéricamente todas repiten un mismo modelo con tres dependencias principales: el depósito o decantador, donde se almacena y decanta el agua salobre; las zonas de evaporación, en las que por exposición al sol aumenta la concentración salina, y los cristalizadores, donde se alcanza la máxima concentración de cloruros hasta la cristalización de la sal. Todas estas dependencias están comunicadas por medio de un complejo sistema de compuertas y canales a través de los cuales el agua pasa hasta el cristalizador.

3. Esta nueva línea de trabajo, en la que venimos trabajando desde hace unos años, plantea una fuerte problemática interpretativa respecto a la funcionalidad y comprensión espacial de éstos y otras restos similares dispersos por nuestro litoral; problemática a la que estamos en parte dando respuestas desde la perspectiva de un análisis paleotopográfico, paleoclimático y neotectónico del medio costero gaditano. 

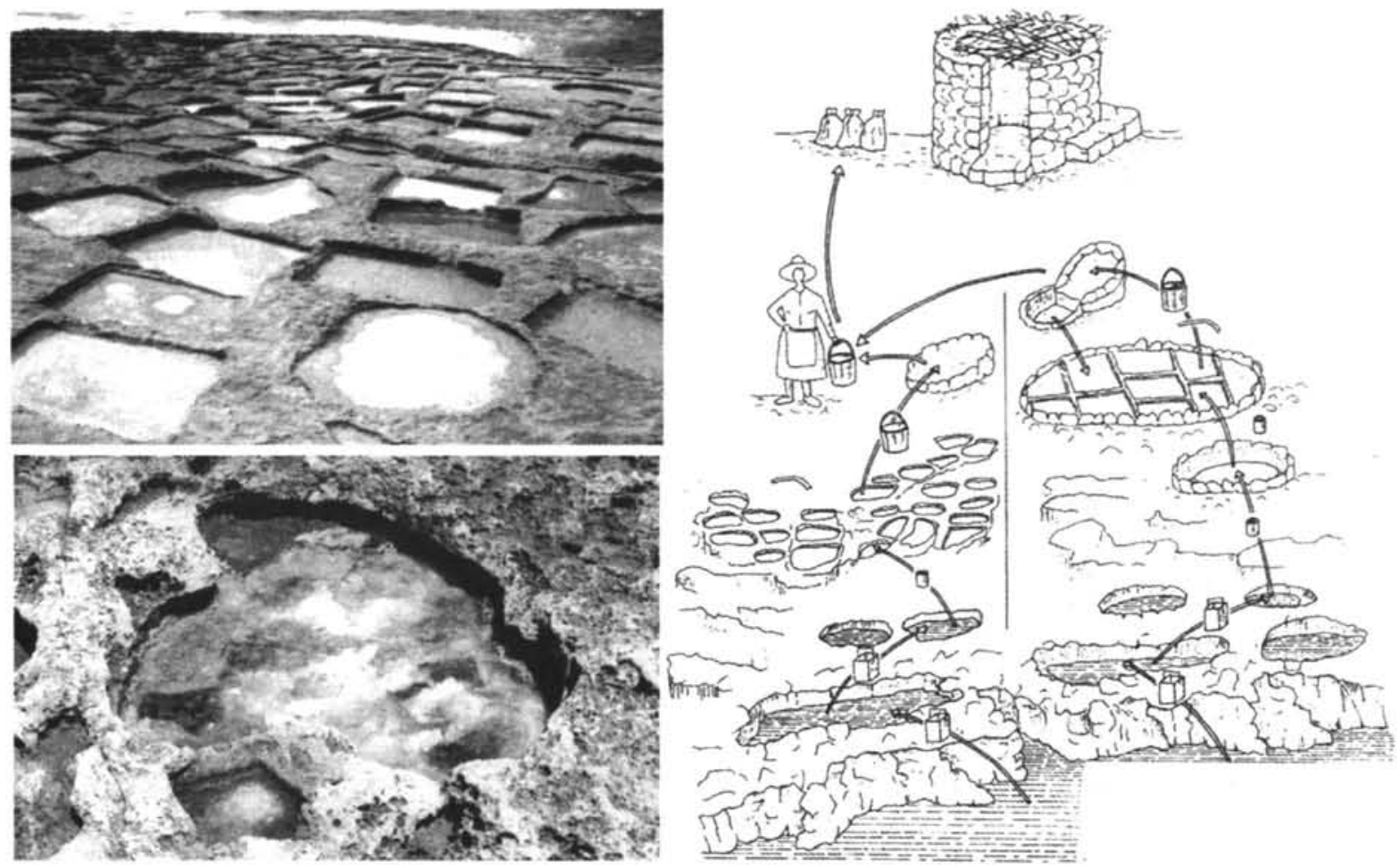

Fig. 1. Aspecto de una explotación de salina sobre roca y esquema de su sistema productivo (Adaptado de Petanidou, T. y Dahm, H., 2002: 149, 151 y 154).

\section{LAS SALINAS DE LA BAHÍA DE CÁDIZ DURANTE LA ANTIGÜEDAD}

La obtención de sal en el marco del territorio gaditano durante la antigüedad debió tener una componente variada aunque no tengamos, por el momento, una amplia constancia arqueológica de ello. Junto a la explotación y comercialización desde el interior de la sal gema y de la obtenida en salinas asociadas a manantiales de aguas salobres (como posiblemente las de la ciudad romana de Iptucci, en el término municipal de Prado del Rey) (Chaves y García 1991), en la costa, como ya se ha apuntado, la sal debió producirse (en función de las necesidades) tanto en salinas sobre roca como sobre fango. Como hemos señalado, y a pesar de la fuerte devastación de las plataformas rocosas costeras gaditanas ligada a su explotación como canteras en Época Moderna, es posible encontrar huellas de las que suponemos antiguas salinas sobre roca. Pero ¿existieron durante la antigüedad, en el marco de las marismas gaditanas, salinas intensivas de evaporación por insolación como las descritas para las proximidades de la propia Roma por Plinio y Namatianus. La respuesta a esta pregunta sólo es posible si se efectúa un análisis geoarqueológico de ese medio físico, encaminado a comprobar la coincidencia espacio-temporal de tres factores fundamentales, a saber:

- La presencia de condiciones naturales adecuadas para la producción de sal.

- La existencia desde antiguo de marismas suficientemente desarrollas como para poder labrar en ellas las salinas.

— La localización de indicadores arqueológicos que evidencien esta actividad. 


\subsection{Las condiciones naturales}

Por lo que respecta a las condiciones naturales se puede afirmar que, desde muy antiguo, el marco de la bahía gaditana se conformaba como un gran humedal con marismas desarrolladas en las que se encajaban el río Guadalete y los caños mareales (Alonso et al. 2000), dibujando un paisaje marismeño provisto de inmejorables condiciones climáticas. La presencia de mareas que favorecen la formación de marismas bañadas sólo en pleamar; las condiciones climatológicas de insolación (con una media de 3098 horas de sol al año), temperatura (moderadas por influencia del Atlántico) y precipitaciones (unos $650 \mathrm{~mm} / \mathrm{año}$ concentrada en otoño e invierno); y el viento de levante (viento seco que sopla especialmente durante los meses de primavera-verano), permitían ya entonces como en la actualidad el proceso de evaporación del agua hasta el punto de la cristalización de los cloruros.

El sistema de corrientes mareales de la bahía de Cádiz se vertebra en la actualidad en torno a dos ejes principales: el río San Pedro por el norte de la Bahía y el caño de Sancti Petri por el sur. A través de éstos y de sus innumerables caños mareales, las marismas gaditanas se encuentran bañadas por las aguas del mar durante todo el año en función del ciclo mareal normal. Otras, sin embargo, sólo permanecen inundadas en los momentos de máxima amplitud mareal (las denominadas mareas vivas, cuyo coeficiente mareal llega a alcanzar los 119). Es precisamente este último tipo de terreno marismeño, por su mayor facilidad para el control del régimen de llenado de los esteros de las salinas a través de las compuertas, el tradicionalmente escogido para la implantación de tajos salineros.

\subsection{La existencia de marismas antiguas}

Los indicadores geoarqueológicos conservados en el marco de la bahía gaditana dan testimonio histórico de la incidencia de dos procesos costeros contrapuestos claramente definidos espacialmente: la erosión y la colmatación (Gracia et al. 1999) (Fig. 2,2). A lo largo de los últimos 2.500 años se aprecian dos momentos en los que la colmatación de la Bahía gaditana parece acelerarse. Uno de estos se sitúa entre los siglos IV y II a. C. y el otro hacia el siglo XVI (Alonso et al. 2000). En ambos casos el origen de este proceso debe ponerse en relación tanto en cambios de la tendencia climática, como en el impacto que la actividad humana produjo sobre el medio natural, especialmente por deforestación y roturación de terrenos para el cultivo: Una gran cantidad de suelo vegetal fue a parar a la red hidrográfica, acumulándose en los espacios restringidos de las desembocaduras fluviales.

Las dataciones efectuadas en el episodio de arranque de la flecha litoral de Valdelagrana han permitido conocer que la unidad de cordones situada más hacia el interior posee, al menos, una antigüedad de 2700 años (Zazo y Goy 2000). Por otra parte, la presencia sobre la misma de un posible asentamiento humano perteneciente al Bronce Pleno (1800-1600 a. C.) y la existencia sobre ella de un puente (según un dibujo realizado por Anton Van den Ringaerde en el año 1567) y un camino posiblemente de época romana (Gómez 1997), vienen a ratificar arqueológicamente tanto su antigüedad como su amplio desarrollo para la época romana. Estos datos ponen en tela de juicio una visión bastante extendida en la investigación arqueológica gaditana, según la cual en ese momento la Bahía de Cádiz se configuraba como un amplio espacio abierto entre Puerto Real y El Puerto de Santa María (Rambaud 1996).

En el siglo IX a. C., en el entorno del Poblado de Doña Blanca (El Puerto de Santa María), existía ya un importante asentamiento prerromano cuya actividad económica se relacionaba principalmente con el comercio marítimo entre oriente y occidente. Con anterioridad al siglo III a. C. el espacio próximo a este asentamiento debió ser sin duda navegable; así lo confirma la recuperación mediante sondeos mecánicos de fragmentos de cerámica griega a cinco metros de profundidad, en los niveles de relleno que colmataban una de las posibles ensenadas portuarias de este enclave. El abandono de Doña Blanca a finales del siglo III a. C. parece estar directamente relacionado, precisamente, con la pérdida de navegabilidad de este espacio. 


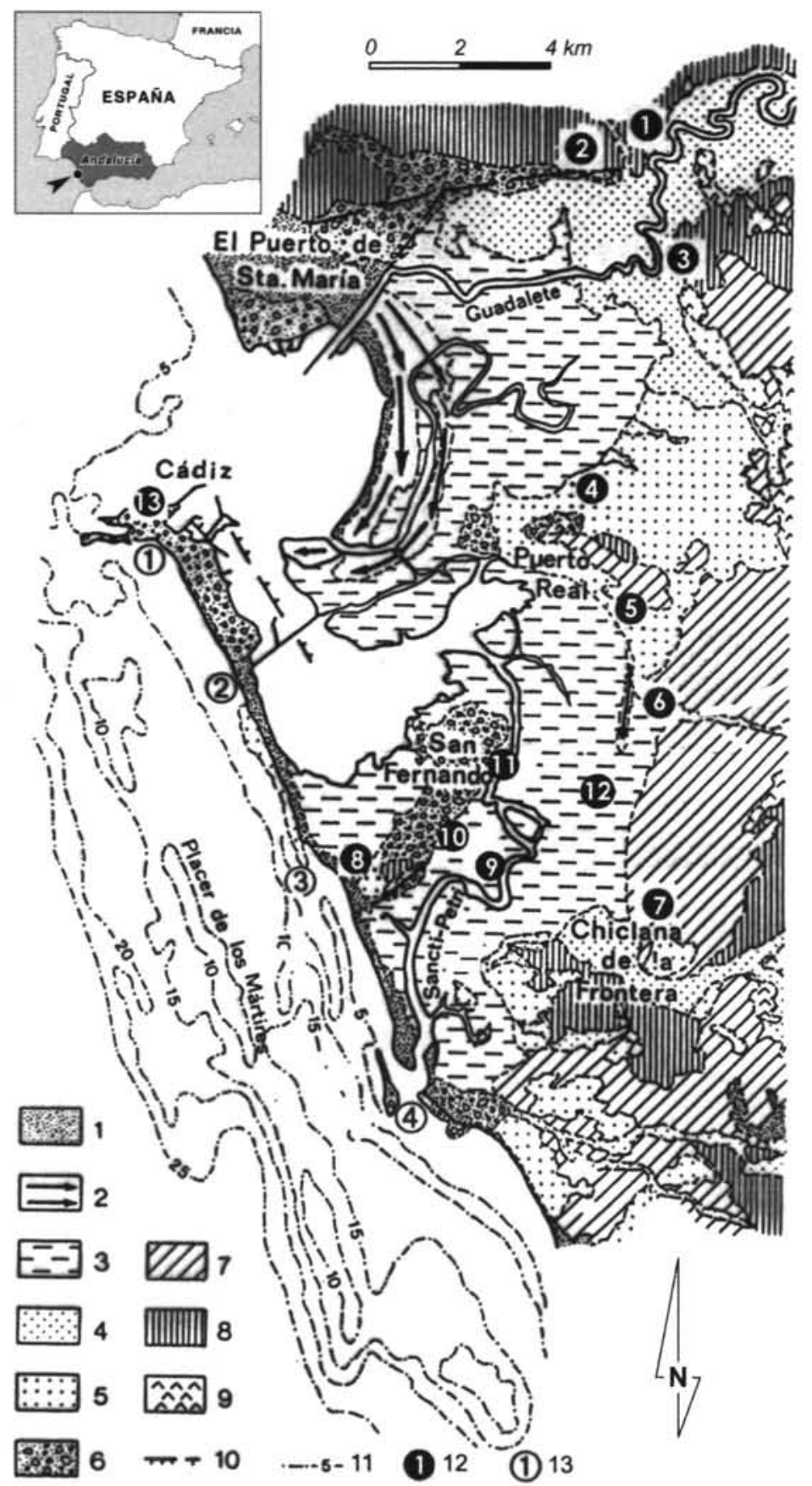

Fig. 2. Mapa geomorfológico de la bahía de Cádiz con indicación de marcadores histórico.-arqueológicos de retroceso y colmatación costera. Leyenda: 1.- Playas y dunas; 2.- Flechas litorales holocenas; 3.- Marismas; 4.- Depósitos fluviales holocenos; 5.- Depósitos pleistocenos; 6.- Depósitos conglomeráticos; 7.- Arenas pliocenas; 8.- Otras unidades terciarias; 9.- Yesos y arcillas triásicas; 10.- Fallas; 11.- Líneas isobatas (en metros); 12.- Indicadores histórico-arqueológicos de colmatación; 13.- Indicadores histórico-arqueológicos de erosión costera. 
Un proceso similar de colmatación hemos podido observar, para inicios del primer milenio a. C. en la zona de Barrio Jarana (Puerto Real), donde se ha caracterizado y datado un cordón litoral, hoy fósil, que estuvo activo y en formación al menos hasta el 900 a. C. (Gracia et al. 1999) (Fig. 2, 6).

Algo más al Sur, en la zona de Camposoto (San Fernando) (González 1998), la existencia de un alfar de ánforas en producción durante los siglos VI y V a. C. y la presencia junto a él de un gran número de ánforas cargadas con pescado salado preparadas para ser exportadas, indica con claridad la proximidad de este emplazamiento a un espacio navegable por el que sacar esos pesados y frágiles contenedores cerámicos. De ello podemos interpretar la escasa colmatación de la zona en ese momento o, al menos, la existencia ya de caños y algún embarcadero que permitiera la aproximación de las embarcaciones hasta la zona de carga.

Tan sólo unos siglos después, la Bahía parece adentrarse en un imparable proceso de colmatación. Hechos, como el de la mayor presencia de biotipos propios de ambientes restringidos procedentes de las actividades de marisqueo en yacimientos como El Castillo de $\mathrm{D}^{2}$ Blanca $^{4}$; el abandono de las anteriores zonas alfareras en la zona de Camposoto; y el desplazamiento de esta actividad industrial en sentido Norte, a lo largo de la costa occidental de San Fernando, así parecen confirmarlo.

En el siglo IV a. C. se produce, efectivamente, el abandono de la producción en los alfares de Camposoto (San Fernando), desplazándose la actividad hacia enclaves próximos a la zona de Torre Alta (apenas $2 \mathrm{Km}$ al norte de la anterior). En esta nueva ubicación la actividad alfarera continúa entre los siglos IV-II a.C., asociada a un nivel de playa hoy fosilizado por marismas (González et al. 1998). Siglos después, en época romana, parece repetirse el mismo proceso, cesando la producción de los talleres de Torre Alta y trasladándose la actividad industrial a otro enclave próximo, más al Norte (zona de Fadrica). El continuo traslado de esta actividad, unido a otros indicadores que posteriormente analizaremos, parece indicar y datar la progradación de esta marisma en sentido Noroeste desde la zona de Camposoto.

En el sector Este, en el espacio comprendido entre los términos municipales de San Fernando, Chiclana de la Frontera y Puerto Real, se localizan igualmente indicadores geoarqueológicos que apuntan el proceso de colmatación de esta zona.

La existencia de una flecha litoral activa al sur de Puerto Real indica con claridad la navegabilidad de esta zona con anterioridad al siglo VIII a. C. (Gracia et al. 1999). Sin embargo, la presencia más al sur de los restos del acueducto romano que suministraba agua a la ciudad de Cádiz, y sus características constructivas (asentado sobre una antigua marisma y cimentado con postes de madera clavados en la misma) ${ }^{5}$, indican claramente la presencia de un espacio de marisma emergida en este espacio hacia el cambio de era (Fig. 2: 12). La colmatación de toda esta zona debió, no obstante, verse fuertemente acelerada precisamente por la construcción del acueducto, especialmente al sur del mismo, zona que desde ese momento quedaría aislada de la influencia mareal. En este sentido, pensamos que toda la franja litoral de la bahía interior, entre Barrio Jarana (Puerto Real) y la desembocadura el río Iro (Chiclana de la Frontera), sería por entonces un espacio restringido de escaso calado y con marismas en formación. No es de extrañar por ello que, si bien toda la zona norte de la Bahía interna (Fig. 2: 4, 3 y 5) presenta una fuerte concentración de alfares romanos para la elaboración de ánforas (García 1998; Lagóstena 1996), este tramo costero carezca de este tipo de instalaciones, presentes sin embargo algo más al interior, en relación con vías de comunicaciones terrestre (Fig. 2: 7).

En la vertiente oriental de la isla de San Fernando encontramos, igualmente, valiosos indicadores al respecto del tema que nos ocupa. Según se aprecia en la cartografía histórica, este espacio fue colmatándose

4. Agradecemos al Prof. Dr. Ruiz Mata la información amablemente facilitada al respecto, cuyos datos se encuentran aún en estudio.

5. Agradecemos a D. Ángel Muñoz, Arqueólogo de la Delegación Provincial de Cultura de Cádiz, los datos facilitados al respecto de la obra y las condiciones en las que se produjo este hallazgo. 
simultáneamente a la progresiva migración del tramo sur del caño de Sancti Petri hacia el SE. En un primer momento (siglo XVI) se aprecia la existencia de una isla en el centro del caño (Fig. 2: 9), justo en la zona donde se produce la confluencia de mareas. En 1734 esta isla se representa ya anexionada a San Fernando por medio de una amplia marisma que colmata el brazo del caño más próximo a la ciudad. Siglos después, este brazo colmatado será nuevamente abierto por el hombre hasta su posterior cegamiento (Cano 1987).

$\mathrm{El}$ análisis microtopográfico de las marismas de la bahía de Cádiz revela que, efectivamente, en este marco existen marismas emergidas a una cota por encima del actual nivel de inundación y desarrollo de marismas activas. Esto podría indicar que en un momento en el que el nivel relativo del mar estaba ligeramente por encima del actual, posiblemente entre el 3000-2200 años a. C., tras el máximo eustático de la transgresión flandriense (Lario 1996), y el 600 a. C., momento en el que según los datos climáticos globales el nivel del mar debía estar ligeramente por debajo del actual (Lario 1996; Gracia et al. 2002). Por su parte, el análisis de imágenes de satélite permite descubrir en el entorno de San Fernando antiguos canales mareales relictos y los niveles de marismas a ellos asociados.

Todo ello viene a confirmar que, desde antiguo, la bahía gaditana era un gran humedal con marismas desarrolladas en las que se encajaban tanto el cauce del río Guadalete como múltiples caños mareales (Fig. 3).

\subsection{Los indicadores arqueológicos}

Estos espacios colmatados fueron utilizados desde el mismo momento de su emersión. La finalidad de ese uso, poco clara aún, debió sin duda estar en relación con diferentes actividades (pesca y marisqueo principalmente) entre las que, por su importancia socioeconómica, figuraba la producción de sal, la cual ha pervivido a lo largo de los siglos hasta la actualidad. Si bien hasta el presente no han estado suficientemente valoradas y a veces mal interpretados, hay que destacar la presencia, en los terrenos inundables de las antiguas salinas desecadas de Camposoto, de alineaciones de escasa altura y reducido espesor... compuestas por restos materiales pétreos, constructivos y cerámicos, tanto púnicos como romanos (especialmente ánforas), reproduciendo el trazado de antiguas estructuras salineras (Fig. 2: 8 y Fig. 4). Estos hallazgos vienen a confirmar que, o bien estamos ante restos de antiguas salinas de época púnico-romana, o bien estos materiales han sido objeto de acarreo desde yacimientos próximos para configurar las estructuras de salinas en un momento posterior. La homogeneidad de los materiales y su contextualización arqueológica parecen indicar más bien lo primero, si bien el nivel de destrucción de estas estructuras no permite un análisis más profundo. Con posterioridad a nuestro estudio, la zona ha sido objeto de atención por otro equipo (Arteaga et al. 2001; Barragán 2001). Al igual que nosotros, llegan a la conclusión de que durante la época romana este sector de San Fernando estaba colmatado y ocupado por el ser humano, si bien difieren en la interpretación de los restos localizados sobre ellas, puestos en relación con estructuras asociadas a centros de producción cerámica.

No son estos los únicos restos existentes de esta índole en la Bahía de Cádiz. Con frecuencia nos llegan noticias de pescadores que, barriendo con artes de arrastre el fondo de algunos caños, recuperan en sus aparejos de pesca ánforas completas de diferentes tipologías. También son muchos los mariscadores que, trabajando en las marismas de San Fernando, han localizado ánforas romanas (e incluso púnicas), en ocasiones aisladas y en otras agrupadas formando alineaciones (Véase símbolo correspondiente en Fig. 3). Uno de estos ejemplos, el único documentado arqueológicamente hasta el momento, es la estructura localizada en la zona denominada de los cargaderos por técnicos del Museo Municipal de San Fernando ${ }^{6}$ (Fig 2: 10).

6. Queremos agradecer a la dirección, técnicos y colaboradores del Museo Municipal de San Fernando (Antonio Sáez y Diego Moreno) la importante información arqueológica y gráfica facilitada. 


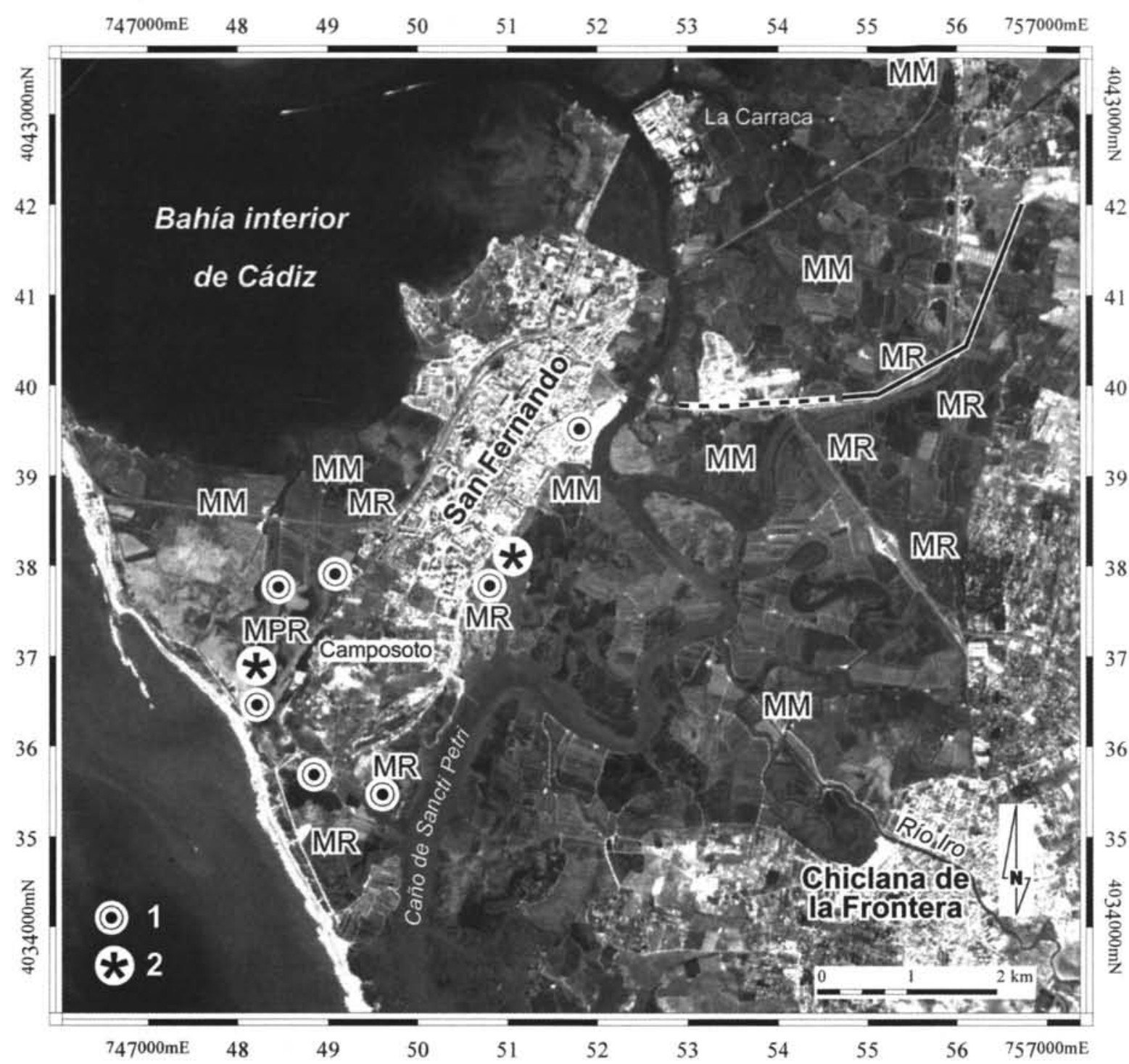

Fig. 3. Localización de indicadores geoarqueológicos de antiguas marismas y salinas en el entorno de San Fernando (Cádiz). Reproducción parcial de la imagen del satélite SPOT 3 (escena 031-276) adquirida en modo pancromático (P) el 14 de noviembre de 1994 a las 11 h $19 \mathrm{mn} 11 \mathrm{~s}$ (TU) y, posteriormente georeferenciada en UTM 29 S E004 (proyección internacional 1924). Leyenda: 1.- Hallazgo de materiales anfóricos (púnicos y romanos) sobre la marismas; 2.- Estructuras salinas romanas; MPR.- Marisma prerromana; MR.- Marisma romana; MM.- Marisma Medieval-Moderna; Alineación.- Trazado del acueducto romano.

Se trata de varias alineaciones de ánforas romanas machihembradas y superpuestas entre sí formando una estructura de 1,5 metros de altura y varias decenas de metros de longitud (Foto 5). Hasta el presente se ha apuntado que podría tratarse de parte de la estructura de un antiguo embarcadero. Sin embargo, su escasa potencia, su situación y emplazamiento (con disposición paralela al caño de alimentación y a la costa, junto a un antiguo canal mareal), su tipología constructiva (sobre una antigua marisma, a base de postes de maderas 


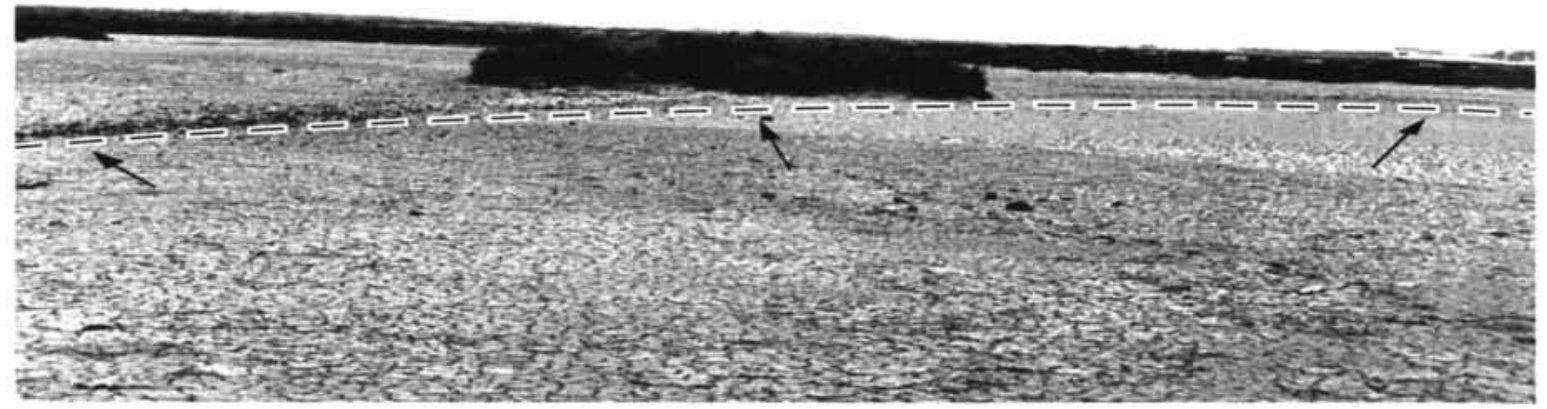

Fig. 4. Huella dejada por un antiguo muro de contención de salina (Camposoto: San Fernando, Cádiz). En el centro (conformando una isleta), la presencia de materiales cerámicos y constructivos de mayor porte ha permitido la mejor conservación de la estructura.

clavados en el fango, piedras y ánforas) y su posición relativa con respecto al nivel del mar actual (inundada en marea alta, sin llegar a quedar totalmente cubierta, y completamente emergida en marea baja), llevan a relacionar esta estructura con la misma función que actualmente cumple, como muro de contención separando espacios interiores de una salina.

Efectivamente, las ánforas han sido habitualmente utilizadas en zonas de marismas para construir estructuras de saneamiento y drenaje y contención del nivel freático (Pesavento 1998), por lo que no es de extrañar que con ese mismo uso fuese empleada en las antiguas salinas gaditanas. No faltan en este sentido en el marco de la Bahía ejemplos de su empleo con este fin en salinas modernas. Por ello, hasta no encontrar una explicación más lógica que permita poner a estas estructuras en relación con otros usos y funciones concretas, interpretamos estos restos como pertenecientes a antiguas salinas de época romana.

Creemos que, tal y como ha sucedido con las salinas de ignición, a lo largo de nuestro litoral se conservan evidencias de antiguas estructuras de salinas de evaporación solar; restos que hasta el presente han sido escasamente valorados o mal interpretados debido, principalmente, a la falta de un análisis multidisciplinar de este medio. Tenemos en este sentido la esperanza de que, en un futuro, el estudio geoarqueológico sistemático de estos hallazgos y su entorno permita arrojar luz sobre una actividad, la salinera, totalmente desconocida para la época antigua.

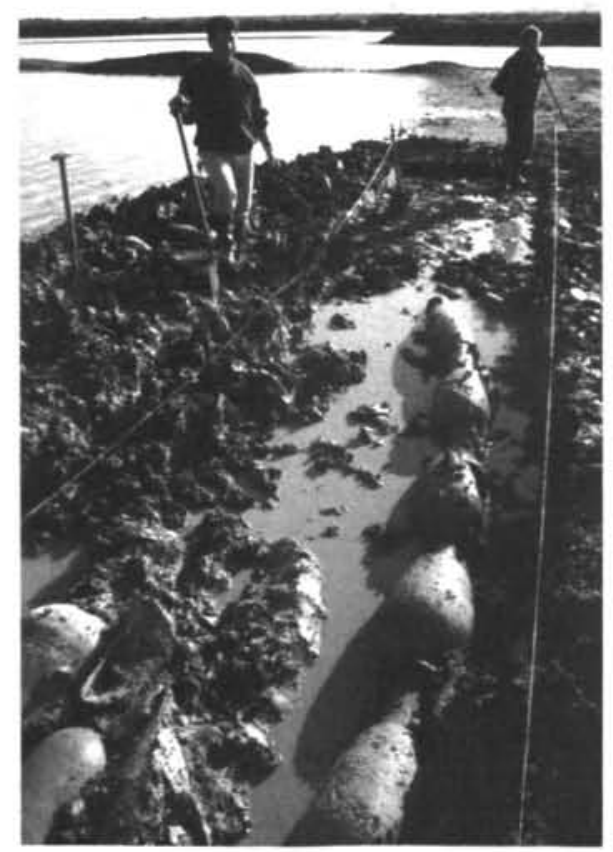

Fig. 5. Parte de un muro de contención del interior de una salina construido con ánforas, piedras y postes de madera clavados en el fango de la marisma romana. 


\section{BIBLIOGRAFÍA}

ALONSO, C.; GALLARDO, M. y MARTÍ, J. et al. (1997): "La sismotectónica de época histórica y su influencia en la Bahía de Cádiz", XXIV Congreso Nacional de Arqueología, volumen 4: 651-660. Cartagena.

ALONSO, C.; GRACIA, F. J. y BENAVENTE, J. (2000): "Las marismas, alfares y salinas como indicadores para la restitución paleotopográfica de la bahía de Cádiz durante la antigüedad", en XVI Encuentros de Historia y Arqueología. Las industrias alfareras y conserveras Fenicio-Púnicas de la Bahía de Cádiz. San Fernando 13-15 de diciembre del 2000. (e.p)

ALONSO, C.; MÉNANTEAU, L.; NAVARRO, M.; MILLE, S. y GRACIA, F. J. (2001): “Antropización histórica de un espacio natural. Las salinas de la Bahía de Cádiz”, PH. Boletín del Instituto Andaluz del Patrimonio Histórico 35: 172-185, Consejería de Cultura. Junta de Andalucía.

ARTEAGA, O.; KÖLLING, A.; KÖLLING, M.; ROOS, A. M.; SCHULZ, H. y SCHULZ, H. D. (2001): "El puerto de Gadir. Investigación geoarqueológica en el casco antiguo de Cádiz", RAMPAS. Revista Atlántica-Mediterránea de Prehistoria y Arqueología Social 4: 345-415.

BARRAGÁN MALLOFRET, D. (2001): Investigación geoarqueológica en San Fernando, Cádiz. Memoria de Licenciatura (inédita), Universidad de Sevilla.

BOAK, M. (1937). "An ordinance of the SALT Merchants at Tebtunis", AMER. Jour of Philology, LVIII: 210-219.

CANO, J. M. (1987). Cartografía de la Isla de León. San Fernando.

CASSEN, S. (2001): "La fabrication du sel. Une hypothèse fonctionnelle pour la forme céramique du caveau de Lannec er Gadouer", en S. Cassen, C. Boujot C \& J. Vaquero (dir.), Éléments d'architecture. Exploration d'un tertre funéraire à Lannec er Cadouer (Erdeven, Morbihan). Constructions et reconstructions dans le Néolithique morbihannais. Propositions pour une lecture symbolique. 2001: 249-265. Publ. Avec le concours Conseil gén. Morbihan, CNRS, Min. Cult., Inst. Cult. Bretagne, Mém. 19.

CHAVES TRISTÁN, F. y GARCÍA VARGAS, E. (1991): "Reflexiones entorno al área comercial de Gades: estudio numismático y económico", Gerión (Homenaje al Dr. Michel Ponsich):139-168. Universidad Complutense.

CHOPIN, J. F. (2002): "Le Collège des Trois-Vallées, Salies-du-Salat (Haute-Garonne). Un nouveau site de production de sel dans le Sud-Ouest de la France", en O. Weller (ed.): Archéologie du sel: Techniques et sociétés. Internationales Archäologie, ASTK 3 Colloque 12.2, XIV Congrès UISPP, Liège, septembre, 2001:189-196.

DAIRE, M.-Y.(dir.) (1994): Le sel gaulois. Bouilleurs de sel et ateliers de briquetage armoriçains à l'Âge $d u$ Fer, Centre Régional d'Archéologie d'Alet, Saint Malo.

ESCACENA CARRASCO, J. L. y RODRÍGUEZ DEZULOAGA MONTESINO, M. (1988): "La Marismilla. ¿Una salina neolítica en el Bajo Guadalquivir?", Revista de Arqueologia 89: 14-24.

ESCACENA CARRASCO, J. L. (1994): “Acerca de la producción de sal en el neolítico andaluz", Actas del encuentro internacional de arqueología del suroeste. Arqueología en el entorno del Bajo Guadiana: 91-118. Huelva.

ESCACENA CARRASCO, J. L.; RODRÍGUEZ DE ZULOAGA MONTESINO, M. y LADRÓN DE GUEVARA SÁNCHEZ, I. (1996): Guadalquivir salobre. Elaboración prehistórica de sal marina en las antiguas bocas del río. Sevilla, Confederación Hidrográfica del Guadalquivir.

ÉTIENNE, R.y MAYET, F. (2002): Salaisons et sauces de poisson hispaniques. Diffusion E. Boccard, París. 
FERNÁNDEZ URIEL, P. (2000): "La industria de la sal”, en Aubet, M. E. y Barthélemy, M. (Eds.) Actas del IV Congreso Internacional de Estudios fenicios y púnicos, vol. II: 345-351. Cádiz, Servicio de Publicaciones de la Universidad de Cádiz.

FRANCOSILVA, A. (s. a.): "Las primeras ordenanzas de el Puerto de Santa María", en El Puertode Santa María entre los siglos XIII y XVI. (S. 1.): 53-78.

FRUTOS REYES, G. y MUÑOZ VICENTE, A. (1996): "La industria pesquera y conservera púnico-gaditana: balance de la investigación. Nuevas perpectivas", Spal 5: 133-165.

GARCÍA VARGAS. E. y MUÑOZ VICENTE, E. (2003): "Reconocer la cultura pesquera de la antigüedad: peces, aparejos pescadores y conservas marinas en la historia antigua de Andalucía", $P H$. Boletín del Instituto Andaluz del Patrimonio Histórico 44. Consejería de Cultura. Junta de Andalucía (e. p.).

GARCÍA VARGAS, E. (1998): La producción de ánforas en la bahía de Cádiz en época romana (siglos II a. $C$. $-I V$ d. C.). Gráfica Sol, Écija

GARCÍA VARGAS, E. y FERRER ALBELDA, E. (2001): "Las salazones de pescado de la Gadir púnica. Estructuras de producción", Laverna 12: 21-41.

GÓMEZ PONCE, C.; BORJA BARRERA, F.; LAGÓSTENA BARRIOS, L.; LÓPEZ AMADOR, J.; RUÍZ GIL, J. A. y BORJA BARRERA, C. (1997): "Primeras fases de la evolución de la flecha de Valdelagrana (El Puerto de Santa María, Cádiz). Datos geoarqueológicos", Cuaternario Ibérico: 165-167.

GONZÁLEZ, B.; TORRES QUIRÓS, J.; HIGUERAS-MILENAS, A. y LAGÓSTENA BARRIOS, L. (2001): "Los inicios de la producción anfórica en la bahía gaditana en época republicana. La intervención de urgencia en la Avda. Pery Junquera (San Fernando, Cádiz)", en Congreso Internacional "Ex Baetica Anphorae", Conservas, aceite y vino de la Bética en el Imperio Romano. (Écija y Sevilla, 17 al 20 de diciembre de 1998): 175-186. Gráfica Sol, Écija.

GONZÁLEZ NAVARRO, J. (1996): Las salinas tradicionales de Gran Canaria. Fedac, Las Palmas de Gran Canaria.

GOULETQUER, P. L. y KLEINMANN, D. (1972): "Les salines historiques et protohistoriques des côtes occidentales de l'Europe", $97^{e}$ Congrès national des sociétés savantes: 17-49. Nantes.

GRACIA, J.; GALLARDO, M.; GILES, F.; ALONSO, C.; MARTÍ, J.; BENAVENTE, J.; REYES, J. y ABAD, E. (1995): "Los niveles holocenos-históricos del Coto de lạ Isleta (Chiclana de la Frontera, Bahía de Cádiz)", Reconstrucción de paloambientes y cambios climáticos durante el Cuaternario. Centro de Ciencias Medioambientales (Serie Monografías, 3): 409-422. Madrid.

GRACIA, F.J.; ALONSO, C.; GALLARDO, M.; GILES, F.; RODRÍGUEZ, J.; BENAVENTE, J. y LÓPEZ AGUAYO, F. (1999): "Aplicación de la geoarqueología al estudio de cambios costeros postflandrienses en la Bahía de Cádiz”, en V. Rosselló (ed.) Geoarqueología i Quaternari litoral. Memorial María Pilar Fumanal: 357-366. Serv. Publ. Univ. Valencia, Valencia.

GRACIA, F. J.; MARTÍNEZ DEL POZO, J. A. y BENAVENTE, J. (2002): "Evolución geomorfológica del entorno de El Retamar", en J. Ramos y M. Lazarich (eds.): Memoria de la excavación arqueológica en el asentamiento del VI Milenio a.n.e. de "El Retamar" (Puerto Real, Cádiz). Consejería de Cultura de la Junta de Andalucía (Serie Monogr. Arqueología, n 3): 15-21. Sevilla.

LAGÓSTENA BARRIOS, L. (1996): Alfarería romana en la bahía de Cádiz. Servicio de Publicaciones de la Universidad de Cádiz. Cádiz.

LARIO GÓMEZ, J.(1996): Último y presente interglaciar en el área de conexión Atlántico-Mediterráneo (Sur de España). Variaciones del nivel del mar, paleoclima y paleoambientes. Tesis Doctoral (inédita), Univ. Complutense de Madrid, 269 pp.

LEMONNIER, P. (1984a): Les salines de l'Ouest. Logique technique, logique sociale., Maison des Sciences de l'Homme \& Presses Univ. Lille. Paris. 
LEMONNIER, P. (1984b): Paludiers de Guérande. Production du sel et histoire économique. (Mém. Inst. d'Ethnologie, XXII), Paris.

MANFREDI, L. I. (1992): "Le saline e il sale nel mondo punico", Rivista di Estudi Fenici XX, 1: 3-14 MORÈRE, N. (1994): "La sal en la Península Ibérica. Los testimonios literarios antiguos", H. Ant. XVIII: 235-250.

MORIN, D. (2002): “L'extraction du sel dans les Alpes durant la Préhistoire. La source dallée de Moriez, Alpes de Haute Provence (France) (cal. B.C. 5810-5526)", en O. Weller (ed.) Archéologie du sel: Techniques et sociétés. Internationales Archäologie, ASTK 3 Colloque 12.2, XIV Congrès UISPP, Liège, septembre, 2001: 153-162.

PASQUINUCCI, M. y MENCHELLI, S. (2002): "The Isola di Coltano Bronze Age village and the salt production in Northcoastal Tuscany (Italy)", en O. Weller (ed.): Archéologie du sel: Techniques et sociétés. Internationales Archäologie, ASTK 3 Colloque 12.2, XIV Congrès UISPP, Liège, septembre, 2001: 177-182.

PASTOR Y RODRÍGUEZ. J.(1880): Estudio sobre el desestanco de la sal y el régimen legal, administrativo y económico más conveniente para la industria salinera española. Imprenta y fundición de M. Tello, Madrid.

PESAVENTO MATTIOLI, S. (ed.) (1998): Bonifiche e drenaggi con anfore in epoca romana: aspetti tecnici e topografici. Atti del seminario di studi, Padova 19-20 ottobre 1995. Franco Cosimo Panini Editore, Modena.

PETANIDOU, T. y DAHM, H. (2002): Salt and salinas as natural resources and alternative poles for local development: The ALAS Project. University of the Aegean (Laboratory of Biogeography and Cultural Ecology, Department of Geography), Mytilene.

RAMBAUD, F. (1996): "Portus Gaditanus. Hipótesis de un nuevo emplazamiento", Revista de Arqueología 187: 24-35.

RAMOS MILLÁN, A. y RIESCO GARCÍA, J. C. (1983): "La villa romana de las canteras (Chipiona). Procesos formativos y transformativos del registro arqueológico e inferencias preliminares del asentamiento", Cuadernos de Prehistoria de la Universidad de Granada 8: 375-417.

RUIZ GIL, J.A. y RUIZ FERNÁNDEZ, J.A. (1987): "Excavaciones de urgencia en el Puerto de Santa María. Cádiz”, Revista de arqueología 74: 5-12.

SÁEZ ROMERO, A. M. (2003): "Control arqueológico de urgencia realizado en el solar ubicado en c/ Albardonero, 24 (San Fernando, Cádiz)”, Anuario Arqueológico de Andalucía (en prensa).

SAILE, T. (2002): "Le commerce du sel dans l'Europe centrale préhistorique", en O. Weller(ed.): Archéologie du sel: Techniques et sociétés. Internationales Archäologie, ASTK 3 Colloque 12.2, XIV Congrès UISPP, Liège, septembre, 2001: 223-231.

SCHUBART, H. (1986): "Hallazgos fenicios y del Bronce Final en la desembocadura del río Guadiaro (Cádiz)", Anuario Arqueológico de Andalucía, II: 200-227.

TASIC, N. (2002): "Sal Trade in the Neolithic of Southeast Europe", en O. Weller (ed.): Archéologie du sel: Techniques et sociétés. Internationales Archäologie, ASTK 3 Colloque 12.2, XIV Congrès UISPP, Liège, septembre, 2001: 147-152.

WELLER, O. (Ed.) (2002a): Archéologie du sel: Techniques et sociétés. Internationales Archäologie, ASTK 3 Colloque 12.2, XIV Congrès UISPP, Liège, septembre, 2001, 257 p.

WELLER, O. (2002b): "Aux origines de la production du sel en Europe. Vestiges, fonctions et enjeux archéologiques", en O. Weller (ed.): Archéologie du sel: Techniques et sociétés. Internationales Archäologie, ASTK 3 Colloque 12.2, XIV Congrès UISPP, Liège, septembre, 2001: 163-175.

ZAZO, C. y GOY, J. L. (2000): "Cambios eustáticos y climáticos durante el cuaternario. Una síntesis sobre su registro en los litorales del sur y sureste peninsular, Islas Canarias y Baleares (España)", en De Andrés, J. R. y Gracia, F. J. (eds.): Geomorfología litoral. Procesos activos. Madrid: 187-206. 